\title{
Molecular Absorption Lines in Galaxies
}

\author{
Tommy Wiklind \\ ESA Space Telescope Division \\ STScI, 3700 San Martin Dr, Baltimore MD 21218, USA
}

\begin{abstract}
Molecular absorption lines have become an important tool in studying the astrochemistry of the dense and cold interstellar medium in both our own Galaxy and high redshift systems. The sensitivity is to first order only dependent on the observed continuum flux. Apart from a few nearby galaxies, molecular absorption lines have been used to study the molecular ISM in 4 galaxies at redshifts $0.25-0.89$. A large number of molecular species and transitions have been observed, allowing a detailed comparison with molecular gas in our own Galaxy. Planned instruments, such as ALMA, will allow studies of a larger number of molecular absorption line systems.
\end{abstract}

\section{Introduction}

The presence of molecules in the interstellar medium (ISM) has been known for almost 70 years. The very first detection of the molecular ISM was done through observations of narrow absorption lines at optical wavelengths, seen towards bright stars in our own Milky Way galaxy (Merrill 1934). Through extensive detective work it was soon established that these lines were due to molecules in interstellar space (Russell 1935; Swings \& Rosenfeld 1937). Today more than 120 molecular species are known to exist in interstellar space. Many of these molecules are complex, containing as many as 13 atoms. These complex species are interesting from an astrochemical point of view, but in order to specify the basic physical and chemical parameters of the molecular gas a smaller set of diagnostic molecules suffice. Knowledge of the physical and chemical properties of the molecular gas allows us to gain an understanding of star formation processes. In the Milky Way these parameters are known through observation of rotational transitions of diagnostic molecular species, radiating at millimeter and sub-millimeter wavelengths.

In external galaxies, however, it becomes increasingly difficult to get a detailed picture of the molecular gas and its physical and chemical properties. This is mainly due to the low covering factor of molecular gas, given the angular resolution provided by existing telescopes, resulting in a low observed signal strength. In addition, some molecules require very high densities and temperatures to be excited and produce observable emission. This situation becomes even more severe for very distant galaxies, where even the best interferometer systems fail to resolve the molecular gas distribution. It is therefore not surprising that the very first detection of molecular gas in external galaxies was done using absorption lines rather than emission lines. With the Parkes $64 \mathrm{~m}$ telescope, Whiteoak \& Gardner (1973) detected $\mathrm{OH}$ in absorption towards the starburst galaxy NGC253. This was followed by detection of additional $\mathrm{OH}$ absorption in NGC4945 (Whiteoak \& Gardner 1975) in the LMC (Whiteoak \& 
Gardner 1976b), and $\mathrm{H}_{2} \mathrm{CO}$ in Centaurus A (Gardner \& Whiteoak 1976) and in the LMC (Whiteoak \& Gardner 1976a).

The first molecule to be observed in emission in external galaxies was CO (Rickards et al. 1975; Solomon \& de Zafra 1975). Since then CO emission has become the standard probe for molecular gas in external galaxies, providing us with pertinent information about the distribution of the molecular gas, its kinematics and the total gas mass. Observation of several different rotational transitions gives a handle on the temperature and density of the molecular component, but little information on the chemical properties of the gas. To achieve the latter, it is necessary to observe additional molecular species, preferably in several different rotational transitions. The sensitivity of today's instruments usually limits this to a few simple molecules in the central regions of the most nearby galaxies.

\section{Molecular Absorption Lines}

The use of molecular absorption lines allows observation of many more molecular species than that possible using the corresponding emission lines. The sensitivity is, to first order, only limited by the observed strength of the background continuum source. The drawback is that the extent of the background source is very limited and only a very small volume of the molecular gas is probed. When using quasars (QSOs) as background sources, the angular extent can be as small as a few tens of $\mu$ arcsec. Another, and more limiting constraint, is the fact that suitable background sources are scarce at millimeter and sub-millimeter wavelengths. Nevertheless, for the most distant objects, molecular absorption lines are the only means available for probing the details of the physical and chemical conditions of the molecular gas.

Molecular absorption occurs whenever the line of sight to a background quasar passes through a sufficiently dense molecular cloud. The molecular gas in nearby galaxies is strongly concentrated to the central regions, making the likelihood for absorption largest whenever the line of sight passes close to the center of an intervening galaxy. Molecular absorption in intervening galaxies is therefore likely to be associated with gravitational lensing.

For optically thin emission the observed property is the emission integrated over velocity, $I_{\mathrm{CO}}$, where: $I_{\mathrm{CO}}=\int T_{\mathrm{a}} d v \propto N_{\mathrm{tot}} T^{-1} e^{-E_{\mathrm{u}} / k T}\left(e^{\mathrm{h} \nu / \mathrm{k} T}-\right.$ 1) $\left[J(T)-J\left(T_{\mathrm{bg}}\right)\right] . N_{\text {tot }}$ is the total column density of a given molecular species, $E_{\mathrm{u}}$ is the upper energy level of a transition with $\Delta E=h \nu, T_{\mathrm{bg}}$ is the local temperature of the Cosmic Microwave Background Radiation (CMBR) and $J(T)=(h \nu / k)\left(e^{h \nu / k T}-1\right)^{-1}$. When $T \rightarrow T_{\mathrm{bg}}$ the signal disappears. For molecular absorption the observable is the velocity integrated opacity $I_{\tau_{\nu}} \propto$ $N_{\text {tot }} T^{-1} \mu_{0}^{2}\left(1-e^{-h \nu / k T}\right) \approx(h \nu / k) N_{\text {tot }} \mu_{0}^{2} T^{-2}$, where $N_{\text {tot }}$ is again the total column density of a given molecular species and $\mu_{0}$ is the permanent dipole moment of the molecule .

\footnotetext{
${ }^{9}$ This expression is strictly speaking only true for linear molecules but represents a reasonable approximation for non-linear molecules as well.
} 
The dependence on $\left(\mu_{0} / T\right)^{2}$ means that the observability of molecular absorption lines increases with the strength of the permanent electric dipole moment and decreasing gas temperature - a situation which to a large extent is the inverse to that of molecular emission. If multiple gas components are present in the line of sight, with equal column densities but characterized by different excitation temperatures, absorption will be most sensitive to the gas component with the lowest temperature. The dependence of the opacity on the permanent dipole moments also means that molecules much less abundant than $\mathrm{CO}$ can be as easily detectable. For instance, $\mathrm{HCO}^{+}$has an abundance which is of the order $5 \times 10^{-4}$ that of $\mathrm{CO}$, yet it is as easy, or easier, to detect in absorption as CO. This is illustrated in Fig. 1, where the observed opacity of the $\mathrm{CO}(1-0)$ and $\mathrm{HCO}^{+}(2-1)$ transitions at $z=0.25$ are compared. In this particular case, the $\mathrm{HCO}^{+}$line has a higher opacity than the $\mathrm{CO}$ line.

Table 1. Properties of molecular absorption line systems.

\begin{tabular}{lccccccc} 
Source & $\begin{array}{c}\mathrm{z}_{\mathrm{a}} \\
\text { (abs) }\end{array}$ & $\begin{array}{c}\mathrm{z}_{\mathrm{e}} \\
\text { (emission) }\end{array}$ & $\begin{array}{c}N_{\mathrm{CO}} \\
\mathrm{cm}^{-2}\end{array}$ & $\begin{array}{c}N_{\mathrm{H}_{2}} \\
\mathrm{~cm}^{-2}\end{array}$ & $\begin{array}{c}N_{\mathrm{HI}} \\
\mathrm{cm}^{-2}\end{array}$ & $\mathrm{AV}$ & $N_{\mathrm{HI}} / N_{H_{2}}$ \\
\hline & & & & & & & \\
Cen A & 0.00184 & 0.0018 & $1.0 \times 10^{16}$ & $2.0 \times 10^{20}$ & $1 \times 10^{20}$ & 50 & 0.5 \\
PKS1413+357 & 0.24671 & 0.247 & $2.3 \times 10^{16}$ & $4.6 \times 10^{20}$ & $1.3 \times 10^{21}$ & 2.0 & 2.8 \\
B3 1504+377A & 0.67335 & 0.673 & $6.0 \times 10^{16}$ & $1.2 \times 10^{21}$ & $2.4 \times 10^{21}$ & 5.0 & 2.0 \\
B31504+377B & 0.67150 & 0.673 & $2.6 \times 10^{16}$ & $5.2 \times 10^{20}$ & $<7 \times 10^{20}$ & $<2$ & $<1.4$ \\
B 0218+357 & 0.68466 & 0.94 & $2.0 \times 10^{19}$ & $4.0 \times 10^{23}$ & $4.0 \times 10^{20}$ & 850 & $1 \times 10^{-3}$ \\
PKS1830-211A & 0.88582 & 2.507 & $2.0 \times 10^{18}$ & $4.0 \times 10^{22}$ & $5.0 \times 10^{20}$ & 100 & $1 \times 10^{-2}$ \\
PKS1830-211B & 0.88489 & 2.507 & $1.0 \times 10^{16(e)}$ & $2.0 \times 10^{20}$ & $1.0 \times 10^{21}$ & 1.8 & 5.0 \\
PKS1830-211C & 0.19267 & 2.507 & $<6 \times 10^{15}$ & $<1 \times 10^{20}$ & $2.5 \times 10^{20}$ & $<0.2$ & $>2.5$ \\
& & & & & & &
\end{tabular}

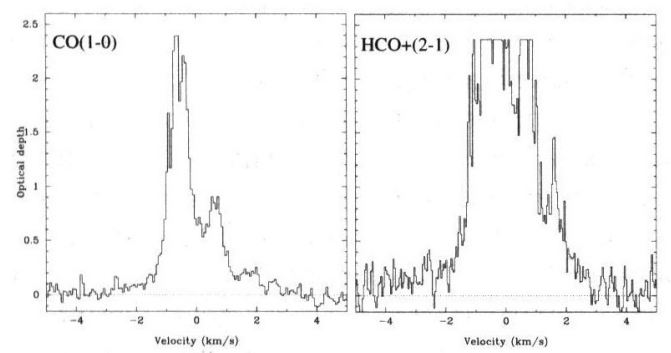

Figure 1. The observed opacity for the $\mathrm{CO}(1-0)$ and $\mathrm{HCO}^{+}(2-1)$ transitions seen at $z=0.25$ towards PKS1413+135. The opacity of the $\mathrm{HCO}^{+}(2-1)$ line is larger than that of the $\mathrm{CO}(1-0)$ line despite of an abundance which is $10^{-3}-10^{-4}$ that of $\mathrm{CO}$. 


\section{Known Molecular Absorption Line Systems}

Local absorption systems Molecular absorption line studies is a relatively new field, both for our own Galaxy and for external galaxies. The small angular extent of QSOs at millimeter wavelengths (tens of $\mu$ arcsec) means that for local molecular clouds, the observed signal is dominated by emission from areas within the telescope beam not covered by the continuum source. It is only with interferometers operating at millimeter wavelengths that it is possible to effectively utilize molecular absorption lines within our own Galaxy. This technique has been used by Lucas \& Liszt in an extended study of the diffuse molecular component in the Milky Way (cf. Liszt \& Lucas 2002; Lucas \& Liszt 2002 and references therein).

Apart from the early observations of $\mathrm{OH}$ and $\mathrm{H}_{2} \mathrm{CO}$ absorption in nearby galaxies, it is only Cen A and the radio galaxy 3C390 that allows absorption line measurements. Both these systems posses a relatively strong flat spectrum AGN. Cen A in particular shows a rich assortment of absorption lines, with several components spread over more than $60 \mathrm{~km} \mathrm{~s}^{-1}$ (cf. Eckart et al. 1990; Israel et al. 1991; Wiklind \& Combes 1997a).

High redshift absorption systems There are four known molecular absorption line systems at higher redshift: $\mathrm{z}=0.25-0.89$. These are listed in Table 1 together with data for the low redshift absorption system seen toward the radio core of Cen A. For the high redshift systems, a total of 22 different molecules have been detected, in 32 different transitions: $\mathrm{CO}, \mathrm{CN}, \mathrm{CS}, \mathrm{OH},(\mathrm{SO}),(\mathrm{LiH})$, $\mathrm{HCO}^{+}, \mathrm{HOC}^{+}, \mathrm{HCN}, \mathrm{HNC}, \mathrm{N}_{2} \mathrm{H}^{+}, \mathrm{H}_{2} \mathrm{O}, \mathrm{H}_{2} \mathrm{CO}, \mathrm{HC}_{3} \mathrm{~N}, \mathrm{C}_{2} \mathrm{H}, \mathrm{C}_{3} \mathrm{H}_{2}, \mathrm{C}^{13} \mathrm{O}$, $\mathrm{C}^{18} \mathrm{O}, \mathrm{H}^{13} \mathrm{CO}, \mathrm{H}^{13} \mathrm{CN}$ and $\mathrm{HC}^{18} \mathrm{O}^{+}$. Molecules in parenthesis are tentative detections. Some of these molecules, $\mathrm{OH}, \mathrm{C}_{2} \mathrm{H}, \mathrm{C}_{3} \mathrm{H}_{2}$ and $\mathrm{HC}_{3} \mathrm{~N}$, are detected at $\mathrm{cm}$ wavelengths (cf. Menten, Carilli \& Reid 1999; Kanekar et al. 2003). As can be seen from Table 1 , the inferred $\mathrm{H}_{2}$ column densities varies by $\sim 10^{3}$. The isotopic species are only detectable towards the systems with the highest column densities.

Two of the four known molecular absorption line systems are situated within the host galaxy to the 'background' continuum source: PKS1413+135 (Wiklind \& Combes 1994) and B3 1504+377 (Wiklind \& Combes 1996b). The latter exhibits two absorption line systems with similar redshifts, $\mathrm{z}=0.67150$ and 0.67335 . The separation in rest-frame velocity is $330 \mathrm{~km} \mathrm{~s}^{-1}$. The two absorption line systems with the highest column densities occur in galaxies which are truly intervening and each acts as a gravitational lens to the background source: B0218+357 and PKS1830-211. In these two systems several isotopic species are detected as well as the main isotopic molecules, showing that the main lines are saturated and optically thick (Combes \& Wiklind 1995; Combes \& Wiklind 1996; Wiklind \& Combes 1996a, 1997b, 1998). Nevertheless, the absorption lines do not reach the zero level, showing that only part of the lensed images are covered by obscuring molecular gas.

Abundance ratios Since most molecules have been observed in two or more transitions, it is possible to determine the excitation temperature and, with the assumption of weak thermal equilibrium, the total column density (cf. Wiklind \& Combes 1997b). The $\mathrm{H}_{2}$ column density remains unknown, but abundance ratios 
between the observed molecular species do not differ from that of corresponding molecular gas in the Milky Way (cf. Wiklind 2003). The abundance of $\mathrm{HCO}^{+}$ is elevated relative to the models of diffuse gas, in the same manner as found for Galactic gas when derived through molecular absorption lines (cf. Wiklind \& Combes 1997b; Lucas \& Liszt 1996).

\section{Special Issues}

Rare transitions Apart from the more common molecular species, such as $\mathrm{CO}$, $\mathrm{HCN}, \mathrm{HCO}^{+}, \mathrm{H}_{2} \mathrm{CO}, \mathrm{N}_{2} \mathrm{H}^{+}$, etc, redshifted molecular absorption lines can also be used to study transitions that have frequencies which normally fall outside transparent atmospheric windows. Such as the ground transition of water vapor, $\mathrm{H}_{2} \mathrm{O}$, LiH and molecular oxygen, $\mathrm{O}_{2}$. The ground transition of $\mathrm{H}_{2} \mathrm{O}$ was first detected at $\mathrm{z}=0.678$ (Combes \& Wiklind 1995), and a tentative detection of $\mathrm{LiH}$ was done in the same system (Combes \& Wiklind 1998). Molecular oxygen, however, remains undetected (Combes \& Wiklind 1995; Combes, Wiklind \& Nakai 1997). In fact, the upper limit to the $\mathrm{O}_{2}$ line achieved through ground based molecular absorption lines is at par with the latest upper limits to the $\mathrm{O}_{2}$ abundance measured with dedicated satellites (Pagani et al. 2003). Both sets of observations suggests that the abundance of $\mathrm{O}_{2}$ in dark clouds is $\sim 10^{-5}$ the predicted abundance from chemical models. Among several different possible explanations to this (cf. Wiklind 2003), the inclusion of grain-reactions into the chemical models appears promising (Roberts \& Herbst 2003).

Future Challenges The main difficulty with using molecular absorption lines in studying the detailed composition of the molecular ISM in external galaxies is that these systems are rare; about 100 times less frequent than damped Lyman$\alpha$ systems. The molecular gas is less extended than other components of the ISM, with a relatively small volume covering factor. Several surveys of potential absorption line systems have so far not found any (see Curran et al. these proceedings and references therein). With planned new instruments, such as ALMA, and a significant increase in the instantaneous wavelength coverage of the receivers, the number of molecular absorption line systems is expected to grow.

\section{References}

Combes F. \& Wiklind T. 1998, A\&A, 334, L81

Combes F., Wiklind T. \& Nakai N. 1997, A\&A, 327, L17

Combes F. \& Wiklind T. 1997, ApJ, 486, L79

Combes F. \& Wiklind T. 1996, in Cold Gas at High Redshift, eds. M. Bremer, P. van der Werf \& C. Carilli, (Kluwer)

Combes F. \& Wiklind T. 1995, A\&A, 303, L61

Eckart A., Cameron, A., Genzel, R., et al. 1990, ApJ, 365, 522

Gardner, F.F. \& Whiteoak, J.B. 1976, MNRAS, 175, 9p

Israel, F.P., van Dishoeck, E.F., Baas, F., de Grauuw, T. \& Phillips, T.G. 1991, A\&A, $245, \mathrm{~L} 13$

Kanekar, N., Chengalur, J.N., de Bruyn, A.G. \& Narisimha, D. 2003, MNRAS, 345, L7

Liszt, H.S. \& Lucas, R. 2002, A\&A, 391, 693 
Lucas, R. \& Liszt, H.S. 2002, A\&A, 384, 1054

Lucas, R. \& Liszt, H.S. 1996, A\&A, 307, 237

Menten, K.M., Carilli, C.L. \& Reid, M.J. 1999, in Highly Redshifted Radio Lines, ASP Conf. Series Vol. 156, eds. C. L. Carilli, K. M. Menten, \& G.I. Langston, p. 218

Merrill, P.W. 1934, PASP, 46, 206

Pagani, L., Olofsson O.A.H., Bergman, P., et al. 2003, A\&A, 403, L77

Rickard, L.J., Palmer, P., Morris, M., Turner, B.E. \& Zuckerman, B. 1975, ApJ, 199, $\mathrm{L} 75$

Roberts, H. \& Herbst, E. 2003, A\&A, 395, 233

Russell, H.N. 1935, MNRAS, 95, 635

Solomon, P.M. \& de Zafra, R. 1975, ApJ, 199, L75

Swings, P. \& Rosenfeld, L. 1937, ApJ, 86, 483

Whiteoak, J.B. \& Gardner, F.F. 1976a, MNRAS, 174, 51p

Whiteoak, J.B. \& Gardner, F.F. 1976b, MNRAS, 176, 25p

Whiteoak, J.B. \& Gardner, F.F. 1975, ApJ, 195, L81

Whiteoak, J.B. \& Gardner, F.F. 1973, Astrophys. Lett., 15, 211

Wiklind, T. 2003, in The Carnegie Centennial Symposium IV: The Formation and Evolution of the Elements, eds. A. McWilliams \& M. Rauch

Wiklind, T. \& Combes, F. 1999, in Highly Redshifted Radio Lines, ASP Conf. Series Vol. 156, eds. C. L. Carilli, K. M. Menten, \& G.I. Langston, p. 202

Wiklind, T. \& Combes, F. 1998, ApJ, 500, 129

Wiklind, T. \& Combes, F. 1997a, A\&A, 324, 51

Wiklind, T. \& Combes, F. 1997b, A\&A, 328, 48

Wiklind, T. \& Combes, F. 1996a, Nature, 379, 139

Wiklind, T. \& Combes, F. 1996b, A\&A, 315, 86

Wiklind, T. \& Combes, F. 1995, A\&A, 299, 382

Wiklind, T. \& Combes, F. 1994, A\&A, 286, L9 